\title{
National trends, complications, and outcomes following transsphenoidal surgery for Cushing's disease from 1993 to 2002
}

\author{
Chirag G. Patil, M.D., Shivanand P. Lad, M.D., Ph.D., Griffith R. Harsh, M.D., \\ EDWARD R. LAWS JR., M.D., AND MAXWELl BOAKYe, M.D. \\ Department of Neurosurgery, Stanford University School of Medicine, Stanford, California
}

\begin{abstract}
Object. Information about complications, patient outcomes, and mortality rate after transsphenoidal surgery (TSS) for Cushing's disease has been derived largely from single-institution series. In this study the authors report on inpatient death, morbidity, and outcomes following TSS for Cushing's disease on a national level.

Methods. All patients in the Nationwide Inpatient Sample (NIS) database who had undergone transsphenoidal resection of a pituitary tumor for Cushing's disease between 1993 and 2002 were included in the study. The number of cases per year, length of stay (LOS), and rates of inpatient complications, death, and adverse outcomes (death or discharge to institution other than home) were abstracted. Univariate and multivariate analyses were performed to determine the effects of patient and hospital characteristics on outcome measures.

Results. According to the NIS, there were an estimated 3525 cases of TSS for Cushing's disease in the US between 1993 and 2002. During this period, there was a trend toward a small increase in the number of TSSs for Cushing's disease. The in-hospital mortality rate was $0.7 \%$, and the complication rate was $42.1 \%$. Diabetes insipidus (15\%), fluid and electrolyte abnormalities (12.5\%), and neurological deficits $(5.6 \%)$ were the most common complications reported. Multivariate analysis showed that complications were more likely in patients with preoperative comorbidities. Patients older than 64 years were much more likely to have an adverse outcome (odds ratio [OR] 20.8) and a prolonged hospital stay (OR 2.2). Women were less likely than men to have an adverse outcome (OR 0.3). A single postoperative complication increased the mean LOS by 3 days, more than tripled the odds of an adverse outcome, and increased the hospital charges by more than US $\$ 7000$.

Conclusions. The authors provided a national perspective on trends, inpatient complications, and outcomes after TSS for Cushing's disease in the US. Postoperative complications had a significantly negative effect on LOS, adverse outcome, and resource utilization. Advanced age and multiple preoperative comorbidities were identified as important risk factors, and their effects on patient outcomes were quantified. (DOI: 10.3171/FOC-07/09/E7)
\end{abstract}

\section{Key Words • complication • Cushing's disease • national database • outcome • pituitary tumor • transsphenoidal surgery}

$\mathrm{C}$ USHING's disease results from cortisol excess caused by the secretion of corticotropin (ACTH) by tumors of the pituitary gland. The disease was first described by Harvey Cushing in $1932 .{ }^{3}$ It has an incidence between 0.7 and 2.4 cases/million persons/yr, and approximately $14 \%$ of pituitary adenomas are ACTH secreting. ${ }^{9}, 14$ Untreated, Cushing's disease leads to hypertension, obesity, diabetes mellitus, osteoporosis, depression, and cognitive deficits. ${ }^{10}$ The treatment of choice in patients with Cushing's disease is a transsphenoidal adenomectomy.

\footnotetext{
Abbreviations used in this paper: $\mathrm{ACTH}=$ adrenocorticotropic hormone; AHRQ = Agency for Healthcare Research and Quality; $\mathrm{CI}=$ confidence interval; $\mathrm{DI}=$ diabetes insipidus; ICD-9 $=$ International Classification of Diseases, 9th Revision; LOS = length of stay; NIS = Nationwide Inpatient Sample; OR = odds ratio; TSS = transsphenoidal surgery.
}

The practice of evidence-based medicine for diseases in which surgery is an option requires knowledge of the benefits and risks of a surgical procedure. Quantifying complication rates and various postoperative outcomes is critical to this risk/benefit calculus. Information about the surgical treatment of Cushing's disease has been derived from single-institution series. ${ }^{4,6,11,12}$ Given that surgical outcomes reported from an individual institution may not be representative of those achieved throughout the country, it is extremely important to carefully examine such outcomes on a national level.

Utilizing data from a representative sample of US hospitals, we analyzed outcomes following TSS for Cushing's disease. We evaluated the national trend for the frequency of TSS for Cushing's disease, the nature and rates of complications, and the frequency of various outcomes such as duration of hospitalization, hospital charges, discharge to a 
C. G. Patil et al.

TABLE 1

Patient and hospital characteristics and associated morbidity following TSS for Cushing's disease in the US from 1993 to 2002

\begin{tabular}{|c|c|c|c|}
\hline Characteristic & No. of Patients (\%) & $\%$ of Patients w/ Complication & $\mathrm{p}$ Value \\
\hline age (yrs) & & & 0.5 \\
\hline$<18$ & $147(4.2)$ & 37.5 & \\
\hline $18-44$ & $2246(63.8)$ & 28.8 & \\
\hline $45-64$ & $966(27.5)$ & 32.0 & \\
\hline$>64$ & $161(4.6)$ & 40.6 & \\
\hline $\operatorname{sex}$ & & & 0.3 \\
\hline female & 2871 (81.6) & 31.1 & \\
\hline male & 649 (18.4) & 28.2 & \\
\hline race & & & 0.5 \\
\hline white & $1930(76.0)$ & 31.7 & \\
\hline black & $225(8.8)$ & 39.1 & \\
\hline other & $385(15.2)$ & 30.4 & \\
\hline comorbidities & & & 0.056 \\
\hline 0 & $1176(33.4)$ & 24.0 & \\
\hline 1 & $1098(31.2)$ & 32.1 & \\
\hline 2 & 798 (22.7) & 37.1 & \\
\hline$\geq 3$ & $448(12.7)$ & 33.3 & \\
\hline hospital volume & & & 0.4 \\
\hline very low & $925(26.3)$ & 27.6 & \\
\hline low & $641(18.2)$ & 32.4 & \\
\hline medium & $718(20.4)$ & 28.7 & \\
\hline high & $677(19.2)$ & 25.2 & \\
\hline very high & $559(15.9)$ & 25.2 & \\
\hline hospital size & & & 0.8 \\
\hline small & $226(6.4)$ & 27.7 & \\
\hline medium & $422(12.0)$ & 30.3 & \\
\hline large & $2872(81.6)$ & 30.9 & \\
\hline treatment period & & & 0.3 \\
\hline $1993-1997$ & $1450(41.2)$ & 33.1 & \\
\hline 1998-2002 & $2070(58.8)$ & 28.7 & \\
\hline
\end{tabular}

facility other than home, and death. We also analyzed the effects of patient and hospital characteristics on these outcome measures.

\section{Clinical Material and Methods}

\section{Data Source}

Data for the years from 1993 to 2002 were obtained from the NIS hospital discharge database. The NIS is maintained by the AHRQ and represents the largest all-payer inpatient care database in the US. It contains data from approximately 8 million discharges annually from 1004 hospitals located in 37 states. $^{7}$ These discharges represent approximately $85 \%$ of all nonfederal hospital discharges in the US. Additional information about the NIS is available at http:// www.ahcpr.gov/data/hcup/nisintro.htm.

\section{Inclusion Criteria}

All patients from 1993 to 2002 with a diagnosis of Cushing's disease (ICD-9 code 255.0) and who had undergone TSS (ICD-9 procedure codes 07.14, 07.62, and 07.65) were included in the present study.

\section{Patient and Hospital Characteristics}

Independent variables such as patient age, sex, race, and comorbidities as well as hospital size and year of treatment were abstracted from the NIS database. Age was stratified into four intervals: younger than 18,18 to 44,45 to 64 , and younger than 64 years. Race was categorized as white, black, and other. The year of treatment was classified into two groups: 1993 to 1997 and 1998 to 2002. The frequencies of medical comorbidities as described and listed by Elixhauser et al..$^{5}$ were calculated using the AHRQ software (http//www.hcup.-us.ahrq.gov/tools_software.jsp; last accessed 06/10/2007). A single comorbidity score was then tabulated and used in the analysis.

Using the unique hospital identifier for each hospital, the annual hospital volume was abstracted. Annual hospital volume was then divided into quintiles representing very low, low, medium, high, and very high volumes. The numeric thresholds for volume were two cases/year (very low), five cases/year (low), 14 cases/year (medium), 23 cases/year (high), and more than 23 cases/year (very high).

\section{Outcome Variables}

The primary outcomes were adverse outcome and complication rate. Adverse outcome was defined as death or discharge to an institution other than home. Complications were classified using the following ICD-9 codes: postoperative neurological complications (997.00-997.09), pulmonary complications (518.81-518.85 and 997.3), thromboembolic complications including deep venous thrombosis and pulmonary embolism $(415,387,415.11-415.19$, 451.0-451.9, and 453.0-453.9), cardiac complications (410 and 997.1), urinary and renal complications (584 and 997.5), hemorrhage or hematoma complicating a procedure (998.1-998.13), DI (253.5), fluid and electrolyte abnormal- 
TABLE 2

Summary of the number of cases and patient outcomes after TSS for Cushing's disease from 1993 to 2002 in the US

\begin{tabular}{lc}
\hline \hline \multicolumn{1}{c}{ Parameter } & Value \\
\hline total no. of cases (discharges) & 3525 \\
\% inhospital deaths & 0.7 \\
total complication rate & 42.1 \\
\% patients w/ $\geq 1$ complication & 30.6 \\
\% patients w/ adverse outcome* & 2.9 \\
mean LOS (days) & 5.5 \\
median LOS (days) & 4.0 \\
mean total charges (US \$) & 25,732 \\
\hline
\end{tabular}

* Adverse outcome was defined as an in-hospital death or discharge to an institution other than home.

ities (276.0-276.9), iatrogenic panhypopituitarism (253.7), and cerebrospinal fluid rhinorrhea (349.81). The number of discharges, deaths, LOS, and hospital charges were also abstracted from the NIS dataset. United States census data were used to adjust population estimates per $1,000,000$ of the US population.

\section{Statistical Analysis}

Categorical outcomes were compared using the chisquare analysis. Probability values less than 0.05 were accepted as significant. A multivariate logistic regression model was constructed for the multivariate analysis; only those variables that satisfied the screening cutoff of $p<0.1$ on univariate analysis were incorporated into the multivariate analysis. The multivariate ORs are reported with the 95\% CIs. For multivariate analysis, the LOS was categorized into normal ( $\leq 75$ th percentile LOS of 6 days) and prolonged LOS ( $>75$ th percentile LOS of 6 days). Multivariate analysis was performed for the following binary outcome variables: complications, adverse outcome, and LOS. Extrapolations to the entire US population were performed using the SAS PROC SURVEY methodology. All data were analyzed using SAS, version 9.1 (SAS Institute, Inc.) running on Windows XP Pro (Microsoft Corp.).

\section{Results}

Three thousand five hundred twenty-five patients were estimated as having undergone TSS for Cushing's disease
TABLE 3

Inpatient complications following TSS for Cushing's disease*

\begin{tabular}{llc}
\hline \hline \multicolumn{1}{c}{ Complication } & Frequency & Complication Rate \\
\hline DI & 528 & 15.0 \\
fluid \& electrolyte abnormality & 441 & 12.5 \\
postop neurological symptom & 197 & 5.6 \\
postop bleeding or hematoma & 93 & 2.6 \\
pulmonary sign & 59 & 1.7 \\
cerebrospinal fluid leak & 48 & 1.4 \\
urinary or renal symptom & 28 & 0.8 \\
diplopia, ptosis, or CN III, IV, & 24 & 0.7 \\
or VI deficit & & \\
cardiac symptom & 24 & 0.7 \\
thromboembolic disease (DVT + PE) & 24 & 0.7 \\
iatrogenic panhypopituitarism & 17 & 0.5 \\
infection & 15 & 0.4 \\
\hline
\end{tabular}

* Note that complication data were available in 3520 cases and that some patients had more than one complication per category. $\mathrm{CN}=$ cranial nerve; $\mathrm{DVT}=$ deep venous thrombosis; $\mathrm{PE}=$ pulmonary embolism.

in the US between 1993 and 2002 (Fig. 1). A small increase in the number of cases between 1993 and 2002 was noted. Patient and hospital characteristics are outlined in Table 1. Patients in the age group from 18 to 44 years represented more than $60 \%$ of the study population. Fewer than 5\% of the patients were pediatric ( $<18$ years). Almost $82 \%$ of the patients were female and $76 \%$ were Caucasian. Thirtythree percent of patients had no preoperative comorbidities, and the majority of patients $(81.6 \%)$ underwent TSS for Cushing's disease at a large hospital. The total inhospital complication rate was $42.1 \%$, and $30.6 \%$ of the patients had at least one complication (Table 2). The inhospital mortality rate was $0.7 \%$. The mean and median LOSs were 5.5 and 4.0 days, respectively. The LOS of patients after TSS for Cushing's disease declined significantly from 1993 to 2002 (Fig. 2).

Table 3 features the frequencies of 10 classes of complications: DI, fluid and electrolyte abnormalities, iatrogenic panhypopituitarism, pulmonary complications, postoperative hemorrhage/hematoma, thromboembolic signs, renal/ urinary complications, cardiac symptoms, infection, and postoperative neurological signs. Diabetes insipidus (15\%) and fluid and electrolyte abnormalities $(12.5 \%)$ were the most frequent symptoms. Neurological complications occurred in 5.6\% of patients and postoperative hemorrhage

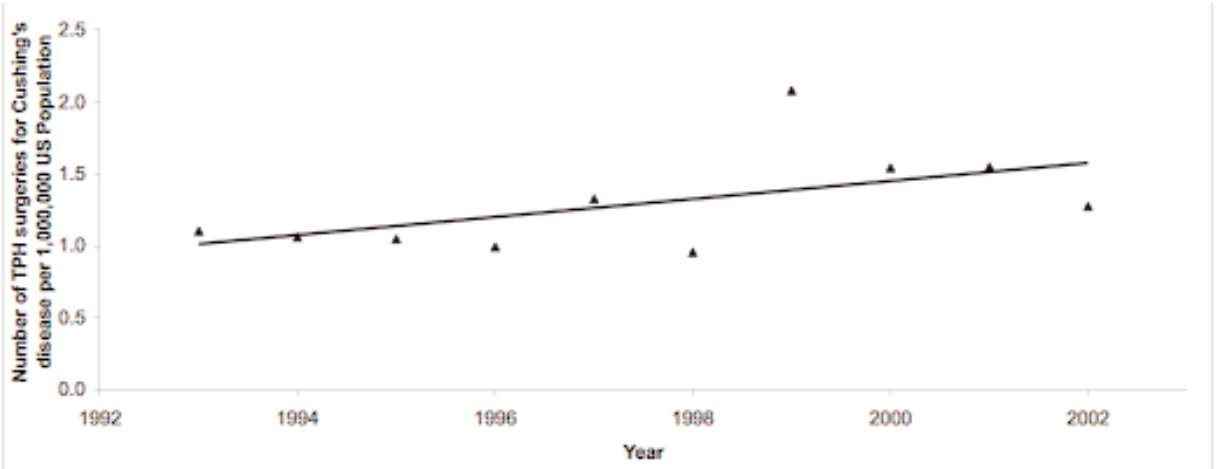

FIG. 1. Graph depicting the population-adjusted number of TSSs performed in the US for Cushing's disease per million persons per year between 1993 and 2002. TPH = transsphenoidal. 
TABLE 4

Effect of complications on mortality rate, LOS, and hospital charges

\begin{tabular}{cccc}
\hline \hline $\begin{array}{c}\text { No. of } \\
\text { Complications }\end{array}$ & $\begin{array}{c}\text { Mean LOS } \\
\text { (days) }\end{array}$ & $\begin{array}{c}\text { \% Patients w/ } \\
\text { Adverse Outcome }\end{array}$ & $\begin{array}{c}\text { Mean Total } \\
\text { Charges (US \$) }\end{array}$ \\
\hline 0 & 4.3 & 1.4 & 21,528 \\
1 & 7.3 & 4.3 & 28,617 \\
2 & 8.2 & 2.7 & 28,684 \\
3 & 14.5 & 21.7 & 88,509 \\
\hline
\end{tabular}

or hematoma in $2.6 \%$. The effects of complications on mortality rate, LOS, adverse outcome, and hospital charges are featured in Table 4. With just one postoperative complication, the mean LOS increased by 3 days, the rate of adverse outcome increased from 1.4 to $4.3 \%$, and the hospital charges increased by more than US $\$ 7000$.

Multivariate analysis showed that the odds of a complication were higher in patients with preoperative comorbidities. A single comorbidity increased the odds of a complication 1.6-fold (OR 1.6, 95\% CI 1.01-2.5), and two comorbidities increased the odds of a complication 1.8-fold (OR 1.8, 95\% CI 1.2-2.8). Multivariate analysis revealed age, complications, and sex as significant factors affecting adverse outcome (Table 5). Having a single postoperative complication almost quadrupled the odds of an adverse outcome (OR 3.8). Moreover, women were 0.3 times less likely than men to have an adverse outcome. Multivariate analysis of LOS showed a prolonged hospital stay for older patients, patients with multiple comorbidities, and those with postoperative complications (Table 6). Patients in the 1998 to 2002 treatment group (OR 0.4) were less likely to have a prolonged LOS than were those in the 1993 to 1997 treatment group.

TABLE 5

Multivariate analysis: effect of patient and hospital characteristics on adverse outcome following TSS for Cushing's disease

\begin{tabular}{|c|c|c|}
\hline Variable & $\begin{array}{l}\text { Odds of Adverse } \\
\text { Outcome (OR) }\end{array}$ & $95 \% \mathrm{CI}$ \\
\hline \multicolumn{3}{|l|}{ age (yrs) } \\
\hline$<18$ & $<0.001^{*}$ & $<0.001-<0.0001$ \\
\hline $18-44$ & $\dagger$ & $\dagger$ \\
\hline $45-64$ & $4.2 *$ & $1.2-14.2$ \\
\hline$>64$ & $20.8 *$ & $4.7-92.6$ \\
\hline \multicolumn{3}{|l|}{$\operatorname{sex}$} \\
\hline male & $\dagger$ & $\dagger$ \\
\hline female & $0.34 *$ & $0.1-0.9$ \\
\hline \multicolumn{3}{|l|}{ comorbidities } \\
\hline 0 & $\dagger$ & $\dagger$ \\
\hline 1 & 0.5 & $0.1-2.7$ \\
\hline 2 & 0.9 & $0.2-4.1$ \\
\hline$\geq 3$ & 2.4 & $0.4-13.4$ \\
\hline \multicolumn{3}{|l|}{ complications } \\
\hline 0 & $\dagger$ & $\dagger$ \\
\hline 1 & $3.8 *$ & $1.4-10.0$ \\
\hline 2 & 4.5 & $0.4-47.7$ \\
\hline$\geq 3$ & $20.6^{*}$ & $4.9-86.7$ \\
\hline
\end{tabular}

* Statistically significant value.

$\dagger$ Reference group for comparison.
TABLE 6

Multivariate analysis: effect of patient and hospital characteristics on LOS following TSS for Cushing's disease

\begin{tabular}{lcc}
\hline \hline Variable & Odds of Prolonged LOS (OR) & $95 \% \mathrm{CI}$ \\
\hline age (yrs) & & \\
$<18$ & 0.6 & $0.2-2.0$ \\
$18-44$ & $*$ & $*$ \\
$45-64$ & $2.0 \dagger$ & $1.2-3.1$ \\
$>64$ & $2.2 \dagger$ & $1.0-4.9$ \\
comorbidities & $*$ & \\
0 & 1.2 & $0.7-2.1$ \\
1 & 1.3 & $0.7-2.6$ \\
2 & $2.4 \dagger$ & $1.2-4.8$ \\
$\geq 3$ & $*$ & $*$ \\
complications & $3.9 \dagger$ & $2.3-6.5$ \\
0 & $6.6 \dagger$ & $3.1-14.0$ \\
1 & $16.0 \dagger$ & $6.6-38.7$ \\
2 & & \\
$\geq 3$ & $*$ & $*$ \\
hospital size & $4.5 \dagger$ & $1.4-14.5$ \\
small & $5.1 \dagger$ & $1.8-14.3$ \\
medium & $*$ & $*$ \\
large & $*$ & $0.2-0.6$ \\
treatment group & $0.4 \dagger$ & \\
$1993-1997$ & & \\
$1998-2002$ & &
\end{tabular}

* Reference group for comparison.

$\dagger$ Statistically significant value.

\section{Discussion}

We reported the outcomes of TSS for Cushing's disease between 1993 and 2002 from a nationally representative sample of US hospitals. The present analysis included 3525 admissions. The inhospital mortality rate was $0.7 \%$, and the inpatient complication rate was $42 \%$. Approximately $30 \%$ of cases had one or more postoperative complications during the inpatient hospitalization. Authors of previous single-institution series have reported complication rates from 9 to $51 \% .^{1,8,11,13}$ Given that the two most frequent complications, DI and fluid and electrolyte abnormalities, are often transient and may be delayed, complication rates figured from assessments performed after discharge may differ from those calculated from inpatient observations. Because of the limitations of the NIS dataset, we were unable to identify the fluid and electrolyte abnormalities caused by DI. Hence, in patients with electrolyte abnormalities caused by DI, two separate complications were coded. The use of this method may have overestimated the true complication rate in our analysis. In addition, the $15 \%$ incidence of DI in the current study represents the rate of transient DI and likely is an overestimate of permanent DI. Determining the rate of permanent DI requires an assessment of endocrine function several months after TSS. Given that the NIS contains information on inpatient admissions only and does not track patients after discharge, determining the incidence of permanent DI was not possible.

Utilizing the NIS, Barker et al. ${ }^{2}$ analyzed outcomes after TSS for all types of pituitary tumors. They reported that $26.5 \%$ of patients had one or more complications, with DI and fluid and electrolyte abnormalities occurring in 10.5 and $8.8 \%$ of the patients, respectively. In our analysis of patients with Cushing's disease, 15\% had DI and 12.5\% 


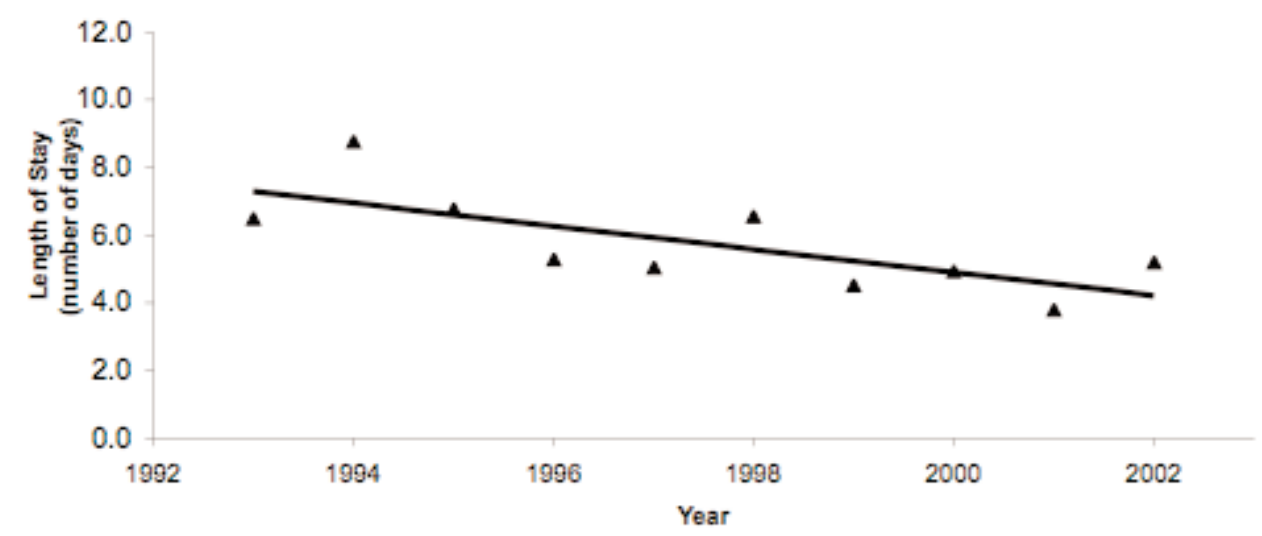

FIG. 2. Graph showing trend for LOS after TSS for Cushing's disease between 1992 and 2002.

had fluid and electrolyte abnormalities. Similarly, patients with Cushing's disease had higher rates of postoperative bleeding (2.6\% compared with $1.6 \%)$ and neurological complications (5.6\% compared with $4.6 \%$ ) than did patients with all types of pituitary tumors. Semple and Laws ${ }^{13}$ have reported a higher post-TSS complication rate for ACTH-secreting tumors than for other pituitary tumors. Our findings also indicated that patients with Cushing's disease may have a higher risk of complications than patients with other pituitary tumors.

National estimates of morbidity, death, and the effect of postoperative complications on resource utilization and outcomes for surgically treated Cushing's disease have not been reported. From a healthcare systems analysis perspective, it is important to quantify the effects of postoperative complications on patient outcomes and resource utilization. We found that just one postoperative complication increases the mean LOS by 3 days, more than triples the odds of an adverse outcome, and increases hospital charges dramatically (Table 4). Therefore, the avoidance, early recognition, and prompt management of complications are extremely important for the efficient surgical care of patients with Cushing's disease.

Comorbidities negatively affect postoperative outcomes in neurosurgery. In our analysis, the results of multivariate logistic regressions demonstrated a significant correlation between the presence of comorbidities and the complication rate. For example, patients with two comorbidities were almost two times more likely to have a complication than those with no comorbidities. Moreover, three or more comorbidities increased the odds of a prolonged hospital stay. Independent of other factors such as comorbidities, advanced age and male sex were correlated with higher rates of adverse outcome and longer LOSs. Patients older than 64 years of age were more than twice as likely to have a prolonged LOS and more than 20 times more likely to have an adverse outcome than those who were 18 to 44 years of age.

Barker and associates ${ }^{2}$ have reported a correlation between surgical outcome and the number of procedures performed annually at a given hospital. Lower complication rates and superior outcomes following TSS for pituitary tumors of all types were noted at high-volume centers. Our analysis of the NIS data for such a volume effect specifi- cally for TSS for Cushing's disease revealed a trend toward lower complication rates at high-volume hospitals, but this trend was not significant, perhaps because of the relatively small sample size.

\section{Conclusions}

In summary, we provided a national perspective on inpatient complications and outcomes following TSS for Cushing's disease in the US. Postoperative complications have a strong undesirable effect on LOS, discharge disposition, and resource utilization. Furthermore, we identified advanced age and preoperative comorbidity as important risk factors for undesirable outcomes, and we quantified their effect on these outcomes.

\section{References}

1. Atkinson AB, Kennedy A, Wiggam MI, McCance DR, Sheridan B: Long-term remission rates after pituitary surgery for Cushing's disease: the need for long-term surveillance. Clin Endocrinol (Oxf) 63:549-559, 2005

2. Barker FG II, Klibanski A, Swearingen B: Transsphenoidal surgery for pituitary tumors in the United States, 1996-2000: mortality, morbidity, and the effects of hospital and surgeon volume. J Clin Endocrinol Metab 88:4709-4719, 2003

3. Cushing $\mathrm{H}$ : The basophil adenomas of the pituitary body and their clinical manifestations (pituitary basophilism). Bull Johns Hopkins Hosp 50:137-195, 1932

4. De Tommasi C, Vance ML, Okonkwo DO, Diallo A, Laws ER Jr: Surgical management of adrenocorticotropic hormone-secreting macroadenomas: outcome and challenges in patients with Cushing's disease or Nelson's syndrome. J Neurosurg 103:825-830, 2005

5. Elixhauser A, Steiner C, Harris DR, Coffey RM: Comorbidity measures for use with administrative data. Med Care 36:8-27, 1998

6. Hammer GD, Tyrrell JB, Lamborn KR, Applebury CB, Hannegan ET, Bell S, et al: Transsphenoidal microsurgery for Cushing's disease: initial outcome and long-term results. J Clin Endocrinol Metab 89:6348-6357, 2004

7. Healthcare Cost and Utilization Project (HCUP) Databases: Nationwide Inpatient Sample. Rockville, MD: Agency for Healthcare Research and Quality, 2007 (http: //www.hcup-us. ahrq.gov/nisoverview.jsp) [Accessed 14 August 2007]

8. Kanter AS, Diallo AO, Jane JA Jr, Sheehan JP, Asthagiri AR, 
Oskouian RJ, et al: Single-center experience with pediatric Cushing's disease. J Neurosurg 103 (5 Suppl):413-420, 2005

9. Kaye AH, Laws ER Jr (eds): Brain Tumors. An Encyclopedic Approach. New York: Churchill Livingstone, 1995

10. Orth DN: Cushing's syndrome. N Engl J Med 332:791-803, 1995

11. Rees DA, Hanna FW, Davies JS, Mills RG, Vafidis J, Scanlon MF: Long-term follow-up results of transsphenoidal surgery for Cushing's disease in a single centre using strict criteria for remission. Clin Endocrinol (Oxf) 56:541-551, 2002

12. Salassa RM, Laws ER Jr, Carpenter PC, Northcutt RC: Transsphenoidal removal of pituitary microadenoma in Cushing's disease. Mayo Clin Proc 53:24-28, 1978
13. Semple PL, Laws ER Jr: Complications in a contemporary series of patients who underwent transsphenoidal surgery for Cushing's disease. J Neurosurg 91:175-179, 1999

14. Simmons NE, Alden TD, Thorner MO, Laws ER Jr: Serum cortisol response to transsphenoidal surgery for Cushing disease. J Neurosurg 95:1-8, 2001

Manuscript submitted June 12, 2007.

Accepted August 9, 2007.

Address correspondence to: Chirag G. Patil, M.D., Stanford Hospital, Department of Neurosurgery, 300 Pasteur Drive, Room R281 MC5327, Stanford, California 94305. email: chiragpatil@ gmail.com. 